\title{
UNVEILING THE STRUCTURE OF NI/NI OXIDE NANOPARTICLES SYSTEM
}

\author{
L. BARRIENTOS ${ }^{\prime}$, S. RODRIGUEZ - LLAMAZARES ${ }^{1}$, J. MERCHANI ${ }^{4}$, P. JARA ${ }^{1}$, N. YUTRONIC ${ }^{1 *}$, V. LAVAYEN $N^{2,3 *}$ \\ ${ }^{I}$ Departamento de Química, Facultad de Ciencias, Universidad de Chile, PO Box 653, Santiago-Chile \\ ${ }^{2}$ Departamento de Física, Universidad Técnica Federico Santa María, Valparaíso 2390123, Chile \\ ${ }^{3}$ Departamento de Química, Facultad de Ciencias Básicas, UMCE \\ ${ }^{4}$ Departamento de Química, Universidad Tecnológica Metropolitana, Av. José Pedro Alessandri 1242, Santiago-Chile
}

(Received: March 6, 2009 - Accepted: July 6, 2009)

\begin{abstract}
This report shows the structural analysis study of the $30-50 \mathrm{~nm} \mathrm{Ni} / \mathrm{Ni}$ oxide nanoparticles obtained by displacement of a nickel-organometallic complex. So using techniques as electron diffraction (ED), and X-ray diffraction it was shown the presence of $\mathrm{Ni}_{2} \mathrm{O}_{3}, \mathrm{NiO}, \mathrm{NiO}_{2}$, and nickel phases on it.
\end{abstract}

Keywords: nickel oxide, nanoparticles, electron diffraction,

\section{INTRODUCTION}

One dimensional nickel $(\mathrm{Ni})$ nanostructures caught the attention for the potential applications as hydrogen storage of Ni nanotubes array and memory devices $^{1}$, as well due to their optical, giant magneto-resistance, and low saturation magnetization behaviour compared with the corresponding bulk materials ${ }^{2,3}$. Mean while, a combination magnetic nanostructure properties with the bio-applications in biomedicine to open new perspectives of the nickel oxide as agent against the leukemia cancer cells ${ }^{4}$.

Recently our group reported the formation of $\mathrm{Ni} / \mathrm{Ni}$ oxides nanoparticles with potential biomedical applications obtained by displacement of a nickelorganometallic complex ${ }^{5}$. However the composition of those nanoparticles are defined as function of the synthesis conditions and not mention in these report. Is noteworthy in an effort to improve the information at this point, to prupose as schaping aim to this work unveling the nickel - based nanoparticles composition to answer in more accurate way, which kind of nickel oxide $\left(\mathrm{Ni}_{2} \mathrm{O}_{3}, \mathrm{NiO}_{2}, \mathrm{NiO}\right)$ is present in the displacement products of the reference ${ }^{5}$.

The better level of understanding in the composition of the nanoparticles is a key issue and takes relevance from the synthetic conditions, uniform shape and size, to their bio-properties behaviour, nonetheless the exploration for new paths research open future applications in this field.

\section{Synthesis}

Nickel nanoparticles termed as $\mathrm{Ni} / \mathrm{NiO}$ oxide-NPs were prepared following the reference elsewhere ${ }^{5}$, so bis $(1,5$ cyclooctadiene $)$ was dissolved in $50 \mathrm{ml}$ of dry THF and mixed with a solution of $0.4 \mathrm{~g}(5.25 \mathrm{mmol})$ of thiourea in anhydrous methanol under $\mathrm{Ar}$ atmosphere at room temperature. The solution was magnetically stirred. After addition of the thiourea solution, the colour of the mixture changed from yellow to brown black (non stabilized Ni-NPs) by decomposition of the organometallic complex. So after centrifugation the nanoparticles changed from dark brown to light brown supernatant.

\section{Samples Characterization}

The morphology of the Ni/Ni oxide-NPs was measured using a transmission electron microscopy, model JEOL 100-SX. Powder X-ray difractograms were recorded in the $2-80^{\circ}$ range on a Siemens D-5000 diffractometer using $\mathrm{Cu}-\mathrm{K \alpha}$

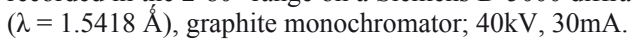

\section{Selected Area Electron Diffraction (SAED)}

The electron diffraction of nanoparticles was obtained using the JEOL microscope internally calibrated with a gold standard (Merck, $99.99 \%$ of purity; $85 \mathrm{kV}, K=4.1614 \mathrm{~cm} \AA$ ). The finely powered samples were dispersed in $n$-hexane and dropped on a conventional carbon-wasted copper grid. The diffraction patterns were obtained using a limiting field aperture $20 \mu \mathrm{m}$. In this way, the diffraction from the grid is avoided and the observed area is minimized.

\section{RESULTS AND DISCUSSION}

Electron Transmission Microscopy

Nickel atoms are released forming readily oxidizable nanoparticles $(\mathrm{Ni} /$
$\mathrm{NiO}$ oxide-NPs) with spherical form, although irregular shapes are also observed ${ }^{5}$, they were not stable at $4^{\circ} \mathrm{C}$; measurements from diffuse reflectance showed that present a semiconductor behaviour $(2.65 \mathrm{eV})$; after $1 \mathrm{~h}$ a brown dark precipitate was formed a precipitate, and the remaining solution became light brown, which indicates that in such a condition aggregation occurred.

$\mathrm{Ni}$ (cyclooctadiene) $)_{2}$ complex was studied combining monocrystal X-ray data with theoretical calculations using the quantum theory of Bader's atoms ${ }^{6}$, it was found that $\mathrm{Ni}$ cannot accept ligand electrons to a great extent, as well the $\pi$ back-donation, which mainly involves $\mathrm{d}(\mathrm{xz})$ and $\mathrm{d}(\mathrm{yz})$ orbitals (orbitals configuration 1, Figure 1), and marginally $d\left(\mathrm{x}^{2}-\mathrm{y}^{2}\right)$, that is slightly hampered by the pseudo-tetrahedral arrangement of ligands (orbitals configuration 2). This agrees with the small $\mathrm{Ni}-\mathrm{C}$ bond orders and the $\mathrm{Ni}$ (cyclooctadiene) ${ }_{2}$ lability.

This means, that there is a poor overlaping among nickel $\delta$ orbitals, and the cyclooctadiene $\pi$ orbitals ligands, as a result an increasing interaction of the $\mathrm{d}$ metal orbital with the $\pi^{*}$ of the ligands, as well the complex is oxidized in air (formation of nickel oxide), and easily undergoes ligand substitution.

The thiourea help in the ligand sustitution of the nickel complex, encouranging the metal releasing, so the oxidation degree is increasing, for that reason was used in our previous report the Cys-PEP ${ }^{5}$ as estabilizer agent. See Figure 1.

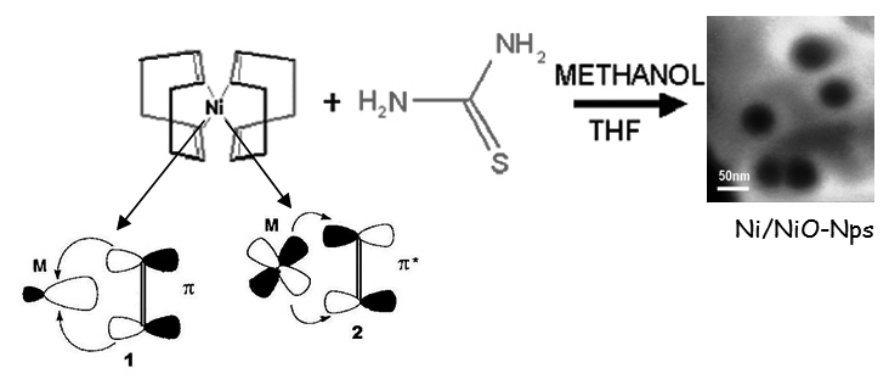

Figure 1: Scheme of the formation of the nickel/nickel oxide nanoparticles, where $\mathrm{M}$ is nickel.

The electron micrograph of this sample (Figure 2) shows the presence of nanoparticles with diameter around $30-50 \mathrm{~nm}$, however the electron diffraction (ED) spot, see Figure 3, shows the presence of preferential orientation in the hexagonal [001] direction of nickel oxide, indeed there were identified two phases, the first of one corresponds to the hexagonal nickel oxide $\left(\mathrm{Ni}_{2} \mathrm{O}_{3}\right)$, see Table I, and the second to rhombohedral nickel oxide $(\mathrm{NiO})$. 


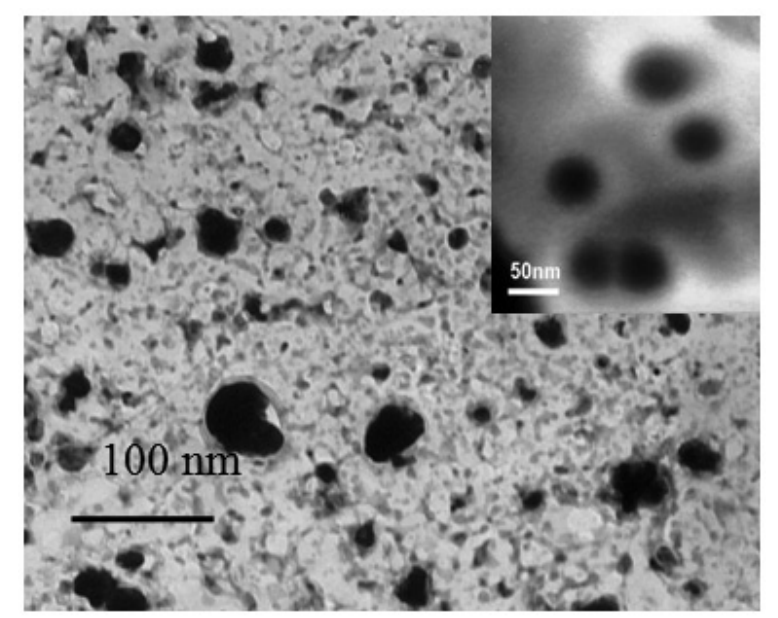

Figure 2: Electron transmission micrograph of the nickel nanoparticles.

Table 1: Phases present in nickel oxide nanoparticles sample.

\begin{tabular}{|c|c|c|}
\hline $\begin{array}{c}d \text {-spacing } \\
{[\mathbf{n m}]}\end{array}$ & Plane & Phase \\
\hline 0.37 & $\{101\}$ & $\mathrm{Ni}_{2} \mathrm{O}_{3}$ \\
\hline 0.20 & $\{002\}$ & $\mathrm{Ni}_{2} \mathrm{O}_{3}$ \\
\hline 015 & $\{400\}$ & $\mathrm{Ni}_{2} \mathrm{O}_{3}$ \\
\hline 0.15 & $\{110\}$ & $\mathrm{NiO}$ \\
\hline 0.14 & $\{104\}$ & $\mathrm{NiO}$ \\
\hline
\end{tabular}

E. I. Alessandrini et al. ${ }^{7}$ described the formation of several phases of nickel oxide by thermal annealing, this procedure showed an epitaxially growing of nickel oxide $(\mathrm{NiO})$ in the [001] direction with the formation of the twinning structure on all four $\{111\}$ planes.

As well thermal annealing can promote the growing of $\mathrm{NiO}$ with the plane $\{100\}$ parallel to the $\{100\}$ of the $\mathrm{Ni}$, twinned with the $\{111\}$ planes $^{7-9}$. This kind of spot's behaviour is similar to our sample, instead of that, the planes observed into the $\mathrm{Ni} / \mathrm{NiO}$ oxide-NPs sample were indexed to two kind of nickel oxides; $\mathrm{Ni}_{2} \mathrm{O}_{3}$ and NiO. See Figure 3 and ED spots of Ref ${ }^{9}$.

The report of A. Mathewa and Y. Shin, shows the formation of flower-like nickel nanocones based of $\mathrm{NiCl}_{2}$, as comparison with the ED spot in Figure 3, it was not observed the reflections from $\{111\},\{200\}$, and $\{220\}$ planes of the crystalline Ni phase ${ }^{10}$.
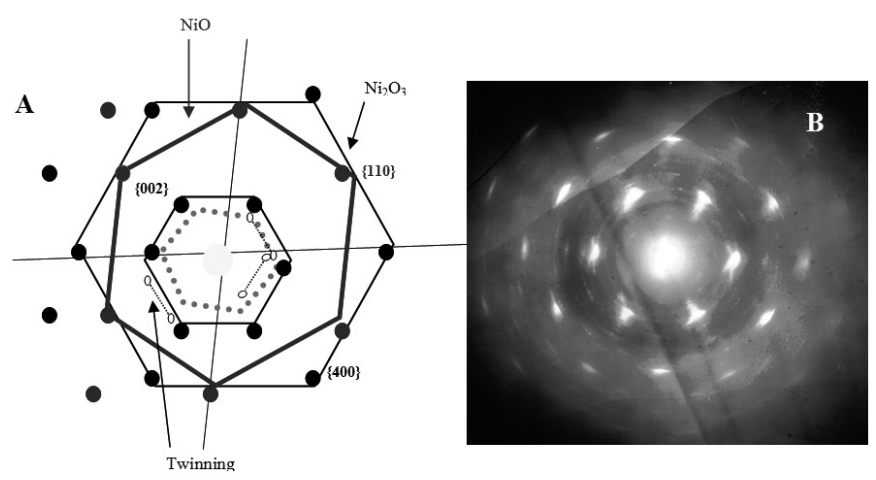

Figure 3: Sheme of the explanation of the different phase that contained the sample (A). Electron diffraction spot of the sample contrained nickel nanoparticles (B).

L.-X. Yanga et. al. reported the SAED pattern of the $\mathrm{Ni}(\mathrm{OH})_{2}$ nanostructures ${ }^{11}$, where the individual nanosheet was single crystalline with thickness along [001] direction (c-axis of hexagonal $\beta$-Ni(OH) $)^{7}$. The spot exhibits bright elongated dots rather than rings - that is the case of the spot
(Figure 3) - indicating that $\mathrm{Ni}(\mathrm{OH})_{2}$ nanosheets are oriented along the [001] direction.

The Table 1 pointing out the planes of the electron diffraction spot (Figure $3)$, so the continuous lines shows the presence of the planes $\{101\},\{400\}$ and the distorted $\{002\}$ plane of the $\mathrm{Ni}_{2} \mathrm{O}_{3}$ and the $\{110\},\{104\}$ of the nickel oxide $(\mathrm{NiO})$, as well the reflections related with the twins that are related with those of the dashed lines (Figure 2).

The micrographic analysis of the nanoparticles show the presence the quite spherical shape, fairly uniform size. However these structures present some aggregated degree because of their dimensions, and high surface energy as same as the nickel structures reported in Ref ${ }^{12}$.

Nonetheless the presence in the ED spot of broadening in the diffraction rings due to tropism of the random small particles; indicates the presence of polycrystalline structure. So electron diffraction reveals that each particle analyzed is composed of small crystal nuclei, which is convincing proof that the particles are aggregated ${ }^{8}$.

As bottom line the Ni-based material present the [001] growing direction, with presence of $\mathrm{Ni}_{2} \mathrm{O}_{3} \mathrm{NiO}$ oxides, as well due to the orientation and level of agregation of the sample, the electron diffraction spot exhibits bright elongated dots, similar to those observed in Refs ${ }^{10-12}$.

\section{X-ray Diffraction}

The X-ray pattern diffraction of the Ni/Ni oxide-NPs compound shows the presence of nickel oxide, taken as reference the cards (JCPDS 89-7129, Ni; 14$0481, \mathrm{Ni}_{2} \mathrm{O}_{3} ; 89-8397 \mathrm{NiO}_{2} ; 89-7131 \mathrm{NiO}$ ). The proposed assignment was done looking for the greatest similarity between the PDF files and the experimental difractograms.

It was not observed "saw-tooth" reflections in the X-ray pattern due to turbostratic phases which are present in ordered two dimensional structure, but whose layers are orientationally disordered.

In the range of $2 \theta: 20-33^{\circ}$ was observed most of the peaks that correspond to the thiourea compound (JCPDS card 18-1968) as the same as reference ${ }^{5}$, as well the pattern at high angles shows the presence of phases that correspond to the $\mathrm{Ni}_{2} \mathrm{O}, \mathrm{NiO}$ oxides, both detected by electron diffraction, nonetheless it was observed the presence of $\mathrm{NiO}_{2}$, and $\mathrm{Ni}$ based phase, see Figure 4 and Table 2.

Table 2: Phases present in the sample contained $\mathrm{Ni} / \mathrm{NiO}$ oxide-NPs.

\begin{tabular}{|c|c|c|c|}
\hline & $\mathbf{2 \theta} /{ }^{\circ}$ & Phase & Indexation \\
\hline $\mathbf{1}$ & 19.38 & $\mathrm{NiO}_{2}$ & $\{001\}^{*}$ \\
\hline $\mathbf{2}$ & 36.25 & $\mathrm{NiO}_{2}$ & $\{111\}$ \\
\hline $\mathbf{3}$ & 50.93 & $\mathrm{NiO}_{2}$ & $\{203\}$ \\
\hline $\mathbf{4}$ & 61.86 & $\mathrm{NiO}_{2}$ & $\{003\}$ \\
\hline $\mathbf{5}$ & 37.07 & $\mathrm{NiO}$ & $\{001\}$ \\
\hline $\mathbf{6}$ & 42.60 & $\mathrm{NiO}$ & $\{111\}^{*}$ \\
\hline $\mathbf{7}$ & 43.85 & $\mathrm{Ni}_{2} \mathrm{O}_{3}$ & $\{100\}$ \\
\hline $\mathbf{8}$ & 51.55 & $\mathrm{Ni}_{2} \mathrm{O}_{3}$ & $\{112\}$ \\
\hline $\mathbf{9}$ & 56.64 & $\mathrm{Ni}_{2} \mathrm{O}_{3}$ & $\{202\}$ \\
\hline $\mathbf{1 0}$ & 65.21 & $\mathrm{Ni}_{2} \mathrm{O}_{3}$ & $\{004\}$ \\
\hline $\mathbf{1 1}$ & 39.25 & $\mathrm{Ni}$ & $\{100\}$ \\
\hline $\mathbf{1 2}$ & 42.65 & $\mathrm{Ni}$ & $\{002\}$ \\
\hline $\mathbf{1 3}$ & 45.34 & $\mathrm{Ni}$ & $\{101\}^{*}$ \\
\hline $\mathbf{1 4}$ & 59.0 & $\mathrm{Ni}$ & $\{102\}$ \\
\hline $\mathbf{1 5}$ & 77.01 & $\mathrm{Ni}$ & $\{103\}$ \\
\hline
\end{tabular}

*strongest reflection of the phase.

Reference Intensity Ratios (RIR) is a standardless method relies that shows a semi-quantitative composition, and is based on the $\mathrm{I} / \mathrm{I}_{\text {cor }}$ values, which are unique to the individual phases and indicate the ability of the material to diffract X-rays with respect to the corundum ${ }^{13}$. The semi-quantitative weight percentage composition is calculated from intensity ratios (available in the PDF database) and the patterns' relative heights. The accuracy of this easyquant method can be improved by taking the integral breadth of peaks from calculated net areas to correct each pattern height ${ }^{13,14}$. 

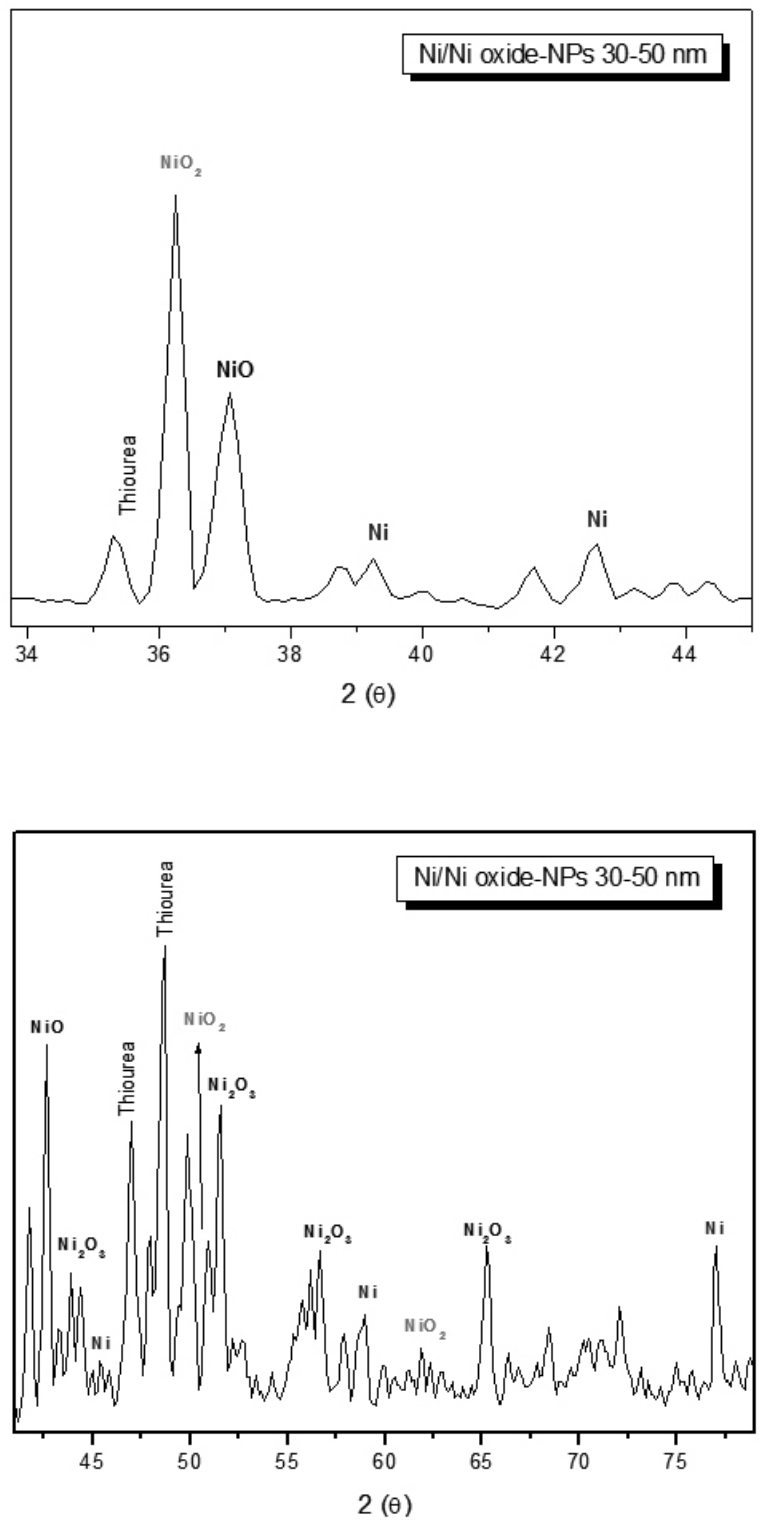

Figure 4: X-ray pattern of the $\mathrm{Ni} / \mathrm{Ni}$ oxide-NPs in the range $35-45^{\circ}$ (upper), and $40-80^{\circ}$ (lower).

The $\mathrm{Ni} / \mathrm{NiO}$ nanoparticles calculated composition was $\mathrm{NiO}(21 \%)$, $\mathrm{Ni}(67 \%), \mathrm{NiO}_{2}(11 \%)$, those values were obtained following the Reference Intensity Ratios (RIR) methodology and taken only the strongest plane diffraction intensities of the phases observed in the X-ray pattern (Table 2).

\section{CONCLUSIONS}

- Through XRD was possible to determine the nature, and crystallinity degree of the nanoparticles, but it was not possible a fully determination the nature (oxidation state) of the phases present on it.

- It was observed from the randomly-oriented microcrystals made of $\mathrm{Ni}$ / $\mathrm{NiO}$ oxide sample by XRD analysis, the presence of the planes $\{001\} \mathrm{NiO}_{2}$, $\{111\} \mathrm{NiO},\{101\} \mathrm{Ni}$, all of them that correspond of those strongest diffracted planes of each nickel phase.

- The crystallinity degree was good in terms of the intensity of the X-ray pattern's peaks, however it ought to take a pattern with high resolution to know in quantitative level the composition of the particles.

- The Reference Intensity Ratios (RIR) method is a good first aproach to know the weight percentage composition of the nanoparticles, indeed the calculated composition is $\mathrm{NiO}(21 \%), \mathrm{Ni}(67 \%), \mathrm{NiO}_{2}(11 \%)$.
- Electron diffraction (ED) technique show the presence of $\mathrm{Ni}_{2} \mathrm{O}_{3}, \mathrm{NiO}$ oxides phases, is noteworthy are inside of the limiting sphere conditions, however were not the same of the X-ray technique, however it acts as complement of thus observed planes by XRD.

- The presence of nickel is detected by XRD and not by electron microscopy, this due to the low resolution TEM equipment used it, and high surface oxidation of nickel by oxygen because due to the high reactivity of the metal released after the substitution of the cyclooctadiene by the thiourea.

- Two techniques, one where a beam of X-rays passed through of randomly-oriented nanoparticles sample crystals produces a pattern (direct space), where the average information to (i) identify the solid material, (ii) crystallinity, and (iii) phase purity can be obtained, and the second technique with punctual analysis capability (inverse space) both acting as complement to show the presence of nickel oxides phase besides the metallic specie.

\section{ACKNOWLEDGEMENTS}

The authors thank the financial contribution of FONDECYT grants (1090282, 1090683, 1090029, 1080505), PBCT grant ACT027, Chile, L.B.P thanks the doctoral scholarship of MECESUP-UMCE, and CONICYT AT24071106 .

\section{REFERENCES}

1. C. Stamm, F. Marty, A. Vaterlaus, V. Weich, S. Egger, U. Maier, U. Ramsperger, H. Fuhrmann, D. Pescia, Science 282, 449, (1998) b) L. Lu M.L. Sui, K. Lu, Science 287, 1463, (2000)

2. H. Wang, X. Jiao, D. Chen, J. Phys. Chem. C, 112, 18793, (2008)

3. K. Sung, S.H. Lee, T.-M. Chung, C. Gyoun Kim, J. Nanosci. Nanotechnol., 8, 4873, (2008)

4. Guo, C. Wu, X. Li, H. Jiang, X. Wang, B. Chen, J. Nanosci. Nanotechnol., 8, 2301, (2008)

5. S. Rodríguez-Llamazares, J. Merchán, I. Olmedo, H.P. Marambio, J.P. Muñoz, P. Jara, J.C.Sturm, B. Chornik, O. Peña, N. Yutronic, M.J. Kogan, J. Nanosci. Nanotechnol., 8, 3820, (2008). b) L. Barrientos, N. Yutronic, F. del Monte, M.C. Gutierrez, P. Jara, New J. Chem., 31, 1400, (2007)

6. P. Macchi, D.M. Proserio, A.Sironi, J. Am. Chem. Soc., 120, 1447, (1998)

7. E. I. Alessandrini, J. F. Freedman, Acta Cryst., 16, 54, (1963)

8. R.M. Hernandez,L. Richter, S. Semancik, S. Stranick, T.E. Mallouk, Chem. Mater., 16, 341, (2004)

9. R.N. Barnett, H.Hkkinen, A.G. Scherbakov, U. Landman, Nano Lett., 4, 1854, (2004)

10. A. Mathewa, N. Munichandraiahb, G.M. Rao, Mat. Sci. Eng. B-Solid, 158, 7 (2009) b) Y. Shin, I.-T. Bae, B.W. Arey, G.J. Exarhos, Mater. Lett., 61, 3215 (2007)

11. L.-X. Yanga, Y.-J. Zhua, H. Tonga, Z.-H. Lianga, L. Lia, L. Zhanga, J. Solid State Chem., 180, 2095 (2007)

12. Z. Wei, P. Yan, W. Feng, J. Dai, Q.Wang, T. Xia, Mater. Charact., 57, 176, (2006)

13. M.C. Mayoral, J.M. Andrés, M.T. Bona, V. Higuera, F.J. Belzunce Surface and Coatings Technol., 202, 5210, (2008)

14. C.R. Hubbard, R.L. Snyder, Powder Diffr., 3, 74, (1988). 